\title{
Consejos Basicos para Diseñar Sistemas Eficientes de Riego ${ }^{1}$
}

\author{
Haimanote K. Bayabil, Kati W. Migliaccio, Michael Dukes, Laura Vasquez, y Carlos Balerdi²
}

\section{Introduccíón}

Los recursos de agua potable están escaseando debido a aumentos en problación y aumentos en la demanda de agua, comida y energía. El estado de Florida proyecta añadir 6 millones de habitantes para el 2030 (Royer and Wang 2017). Además, eventos extremos del tiempo (p.e. inundaciones y sequías) ya son fenómeno común. Por lo tanto a medida que el agua potable escasea y las sequias son frecuentes hay más necesidad de ser eficientes en el uso de recursos hidraulicos. Han habido importantes adelantos en tecnologías de (p.e. válvulas eléctricas, controles inteligentes, sensores de humedad de suelos, etc.) que ahorran agua (Dukes 2012). Sin embargo, la efectividad de estas tecnologías depende de varios factores como el diseño de sistemas de Diseñar equipos y sistemas de eficientes no sólo ahorran dinero pero también ahorran agua.

\section{Factores a Considerar al Diseñar Sistemas de Riego}

Este documento provee un panorama básico de los factores más importantes al diseñar, Figura 1 enseña un esquema de los principales factores en el diseño de un sistema de riego.

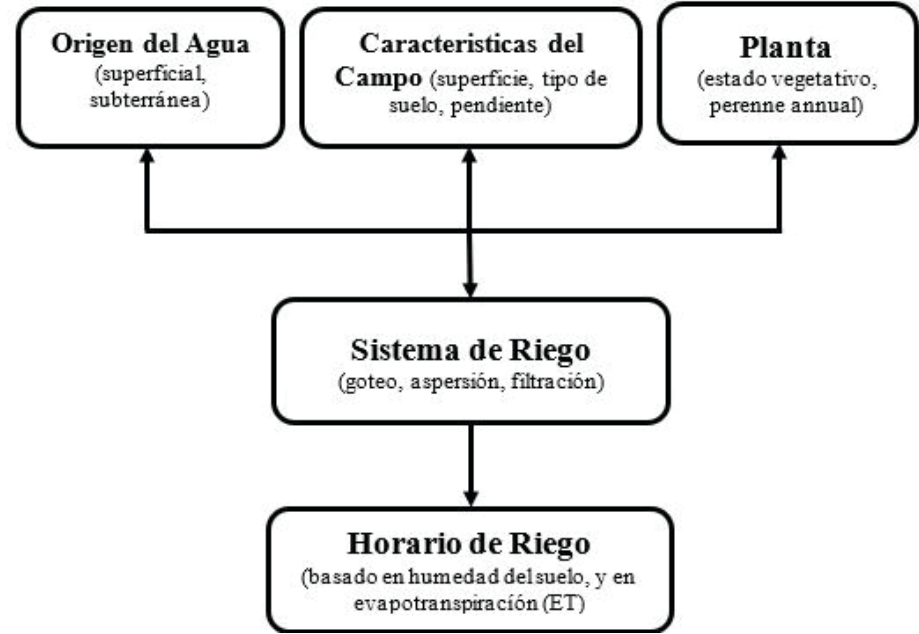

Figura 1. Esquema de los principales factores en el diseño de un sistema de riego.

Créditos: Haimanote K. Bayabil, UF/IFAS

\section{Origen del Agua}

El total de agua potable usada en Florida incluyendo todos los usos es de 6.4 billones de galones por día. Casi dos tercios de ésta agua es subterránea y el resto del agua es superficial (Marella 2015). Casi el 40\% del agua subterrańea se usa en la agricultura y el $36 \%$ es para uso público. El $24 \%$ restante se usa para la generar electricidad, riego de

1. Este documento, AE549, es uno de una serie de publicaciones del Departamento de Ingenieria Agricola y Biologica, Servicio de Extensión Cooperativa de la Florida, Instituto de Alimentos y Ciencias Agrícolas, Universidad de la Florida (UF/IFAS Extension). Fecha de primera publicación: diciembre 2020. Visite nuestro sitio web EDIS en <https://edis.ifas.ufl.edu>. The English version of this document is Basic Tips for Designing Efficient Irrigation Systems (AE539).

2. Haimanote K. Bayabil, assistant professor, Department of Agricultural and Biological Engineering, UF/IFAS Tropical Research and Education Center; Kati W. Migliaccio, chair and professor, Department of Agricultural and Biological Engineering; Michael Dukes, professor, Department of Agricultural and Biological Engineering, and director, Center for Land Use Efficiency; Laura Vasquez, Florida Yards \& Neighborhoods supervisor, UF/IFAS Extension Miami-Dade County; and Carlos Balerdi, tropical fruit crops agent emeritus, UF/IFAS Extension Miami-Dade County; UF/IFAS Extension, Gainesville, FL 32611.

The Institute of Food and Agricultural Sciences (IFAS) is an Equal Opportunity Institution authorized to provide research, educational information and other services

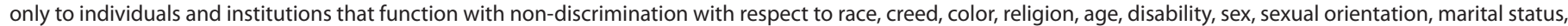

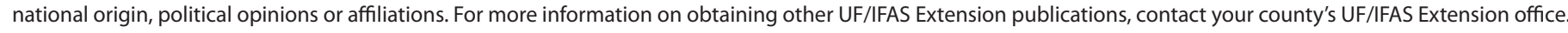
U.S. Department of Agriculture, UF/IFAS Extension Service, University of Florida, IFAS, Florida A \& M University Cooperative Extension Program, and Boards of County Commissioners Cooperating. Nick T. Place, dean for UF/IFAS Extension. 
jardines, recreación, mineria commercial e industrial y el uso autodoméstico. El uso del agua potable en Florida sigue el curso de la densidad de población y de la intensidad de cosechas regadas. El uso de agua potable es mayor en el Condado Palm Beach (más de 1 billon de galones por dia). Conocer el origen, calidad y disponibilidad de agua para riego es crítico. El origen del agua puede ser de agua reciclada, superficial o subterrańea. Dependiendo del origen del uso, la cantidad de agua disponible y el equipo necesario para transpotarla, difiere. La disponibilidad del agua para riego puede estar sujeto a ordenanzas locales, dependiendo de varios factores.

\section{Características del Campo}

Las características del campo (p.e. tamaño, pendiente, tipo de suelo) pueden afectar el tipo de sistema de riego, equipos de riego necesarios (p.e. bomba) y tipos de planta.

\section{El Tamaño del Campo}

El tamaño del campo afecta el máximo número de plantas que pueden sembrarse y por lo tanto el total de riego requerido.or la bomba y la presión original. La pérdida de presión debe considerarse en el diseño de un sistema de riego para minimizar caidas de presión y variaciones en el area regada.

\section{Pendiente}

La pendiente del terreno afecta el flujo del agua y la uniformidad de la distribucón del riego. El agua fluye de alto potencial (alta elevación) a bajo potencial (baja elevación). Dependiendo del trazado del sistema de regadío, los campos con elevaciones más pequeñas pudieran recibir demasiada agua y los de altas elevaciones puedieran recibir muy poca. Bombas se pudieran necesitar para conducir suficiente agua contra una pendiente. Además, la pendiente pudiera promover corrientes y erosión. En algunos casos nivelar los campos se puede considerar.

\section{Propiedades del Suelo}

Las propiedades del suelo (p.e. textura, estructura, profundidad, contenido de materiar orgánica) afectan las relaciones del suelo con la capicidad de retención de agua. La capacidad de retención de agua afecta la frecuencia y la cantidad de agua aplicada. Por ejemplo, los suelos arenosos tienen baja capacidad de retención y necesitan riego más frecuentemente con menos cantidad de agua (Tabla 1). Alternativamente, los suelos arcillosos tienen relativamente más capacidad para retener agua y pudieran regarse menos frecentemente a mayores applicaciones de agua. La profundidad del suelo también afecta la profundidad efectiva de las raices y el lavado de agua y nutrientes por debajo de la zona radical. Hay información general en la ventana Web Soil Survey (https://websoilsurvey.sc.egov.usda.gov/ App/WebSoilSurvey.aspx) generada por el USDA Natural Resources Conservation Service (NRCS). Sin embargo, la seguridad de esa información debe verificarse con medidadas en el campo.

\section{Tipo de Planta}

El tipo de planta afecta la selección del sistema de riego. Los requerimientos de riego difieren dependiendo del estado de crecimiento de la planta. Además los reportes señanlan que las cosechas anuales tienen menores requerimientos de riego comparado a plantas perennes (Vico and Brunsell 2018), diferentes plantas tienen diferentes densidades de siembra (espaciamiento). Si el mismo sistema de riego es usado para regar diferentes plantas, éstas deben ser sembradas en zonas diferentes para que sean regadas independientemente. Los coeficientes $(\mathrm{Kc})$ varían con los estados de crecimiento para difirentes plantas. Así, los valores específicos de plantas Kc deben usarse para calcular los requerimientos actuales de evapotranspiración (ETc) y riego. Los valores de mercado de las cosechas afectan la posibilidad de sistemas de riego más costosos. Cosechas de alto valor economico pueden permitir inversiones mayores en un sistema de riego, ésto puede no ser possible en cosechas de menor valor.

Las siguientes publicaciones documentos contienen información adicional sobre coeficientes de cosechas y requerimientos de riego para ellas.

- Evapotranspiration: Potential or Reference?: https://edis. ifas.ufl.edu/ae256

- Evapotranspiration-Based Irrigation for Agriculture: Crop Coefficients of Some Commercial Crops in Florida: https:// edis.ifas.ufl.edu/ae456

- Evapotranspiration-Based Irrigation Scheduling for Agriculture: https://edis.ifas.ufl.edu/ae457

- Size, Biomass, and Nitrogen Relationships with Sweet Orange Tree Growth: https://doi.org/10.21273/ JASHS.131.1.149

- Principles and Practices of Irrigation Management for Vegetables: https://edis.ifas.ufl.edu/cv107

\section{Tipos de Sistemas de Regadío}

Un sistema de riego distribuye agua de su origen hasta el campo. Identificar el mejor mecanismo de condución para una situación específica es critico. El tipo de planta, 
de suelo, características del jardín, la cantidad de agua, la presión, y el costo afectan la selección del sistema de riego. Esta sección discute los sistemas de riego más importantes (goteo, aspersores y filtración).

\section{Sistema de Riego por Goteo}

Los sistemas de riego por goteo son los más eficientes y es usado en cosechas sembradas en surcos. El sitema de goteo puede ser superficial o subterráneo, y mangueras de polietileno son usadas comunmente. En sistemas de goteo, al agua es aplicada directamente al sistema de raices de la planta usando goteros. Esto reduce pérdidas de agua debido al acarreo superficial y al a lavado por debajo la zona radical. La densidad de siembra guia el espaciamiento de los goteros. Los goteros se colocan en la manguera o los goteros se pueden instalar perforando a la manguera usando las herramientas apropiadas. Los goteros varian en la cantidad descargada (gpm). Esto hace al sistema de goteo apropiado para todos los tipos de suelo. Hay compuestos fisicos (p.e. particulas de arena), biológicos (p.e. bacterias) y químicos que pueden tupir los goteros. Usando una conbinación de estrategias, como instalar filtros, limpieza de mangueras abriendo los terminales, uso de desinfectantes (p.e. cloro) y/o injección de ácidos al sistema, pueden mitigar la tupición de goteros. La Figura 2 enseña un es esquema de los mayores componentes de un sistema típico de goteo.

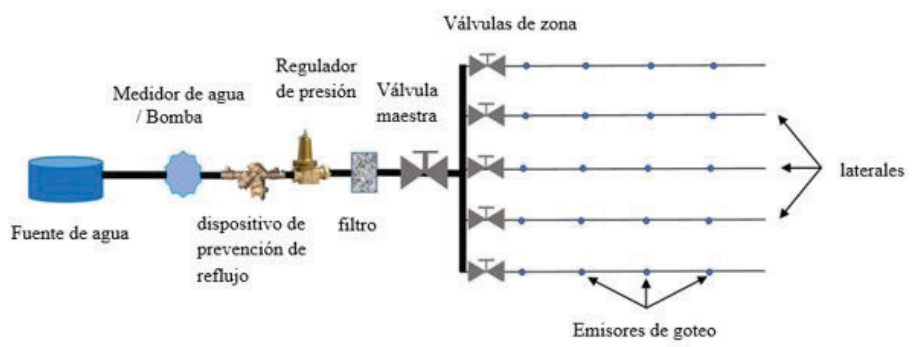

Figura 2. Esquema de un sistema de riego por goteo simplificado. Créditos: Haimanote K. Bayabil, UF/IFAS

\section{VENTAJAS}

- Aplicación localizada en o cerca de la zona de raices

- El sistema de riego más eficiente porque reduce pérdidas de agua resultante del acarreo y percolación profunda

- Puede ayudar a suprimir malezas, especialmente si se combina con colchón plástico

- Distribuye el agua uniformemente

- El agua no contacta a las hojas (p.e. lechuga), reduciendo riesgos de enfermedad

\section{DESVENTAJAS}

- Se daña facilemente por equipos, luz solar, roedores, animales salvajes, etc.
- Nececita ser reemplazado más frecuentemente que otros sistemas

- Propenso a tupiciones; necesita filtración

- Más costoso para grandes áreas

- Requiere mantenimiento frecuente

\section{Sistema de Riego por Aspersión}

En los sistemas de aspersión, el agua se distribuye usando de asperores. Dependiendo de las especificaciones, los regadores de aspersión tienen diferentes radios de aspersión, presiones de operación, y proporciones de aplicaciones. Hay diferentes tipos de sistemas de aspersión tales como área, micro aspersores y rotatorios. Un sistema de aspersión aŕea es a menudo de alta presión con alto volumen de aplicación, donde se aplica el agua por encima de la cosecha. Los aplicadores areos se usan en agricultura, viveros, y en jardines urbanos. Los micro aspersores usan baja presión y volumen de mediano a bajo y se adaptan para cosechas de alto valor como los árboles frutales. Con el sistema de aspersión aérea no es possible regar por encima del follaje de árboles grandes. Los micro aspersores permiten aplicaciones de agua dirigidas al suelo, haciéndolo más eficiente que un sistema de riego de aspersión aérea.

\section{VENTAJAS}

- Duran más que el sistema de goteo

- Se usan en agricultura, patios, jardines y en riego de viveros

- Los aspersores aéreos usan alta presión, y mediano a alto volumen

- Tiene diferentes diseños y ángulos de descarga

- Pueden usarse para protección contra heladas

\section{DESVENTAJAS}

- Menos eficientes que el sistema de goteo

- Riega espacios abiertos entre surcos

- Más susceptible al acarreo por viento que el goteo

- Más potencial de acarreo y erosión que el goteo

- Riesgo de enfermedades porque moja el follaje

\section{Sistema de Riego por Penetración}

En el sistema de riego por penetración, el agua se durige a las raices de las plantas. Este tipo de riego altera el nivel del manto freático. Este sistema es sólo applicable en campos llanos con la precencia de una capa impermeable por debajo de la zona de raices que permite ajustar el nivel del manto freático. Este sistema es común en Florida donde al manto freático y los suelos arenosos están por enzima 
de una capa inpermeable (Rogers et al. 2018; Zotarelli et. al 2019). El sistema por infiltración es uno de los más ineficientes debido al alto volumen de agua necesitado para elevar el nivel del manto freático hasta la zona de raices de la cosecha (Zotarelli et al. 2019). Zotarelli et al. (2019) y Rogers et al. (2018) dan información adicional sobre éste sistema en Florida.

\section{VENTAJAS}

- Se implementa facilmente

- Relativamente bajos costos de operación y mantenimiento

- Menos enfermedades porque el agua no moja al follage

- No hay problemas de tupiciones; no necesita filtración

\section{DESVENTAJAS}

- El sistema menos efectivo

- Sólo se usa en campos llanos con una capa impermeable debajo de la zona de raices que permite ajustes del agua a través del manto freático

- Requiere agua adicional para elevar el manto freático hasta la zona de raices

- Pudiera causar condiciones de saturación de agua si se maneja mal

\section{Hidráulica Básica de Riego}

El diseño de sistemas, de riegos eficientes require un entendimiento de hidráulica de riego, que trata con el flujo de agua a través de un sistema de riego. Varios factores afectan el flujo de agua en un sistema de riego, como la presión, el tamaño de las tuberias, las vávulas, los metros, y los regores o aspersores. Esta sección discute algunos de los factores más importantes que afectan el flujo de agua en un sistema de tuberías.

\section{Presión}

El principio general en riego es que el agua fluye a través de un sistema de riego (p.e. tuberias, válvulas, metros, etc.) debida a energía como la presión. La presión puede ser expresada en diferentes formas, como pies, libras por pulgada cuadrada (psi), bar y pascal. La presión de una columna de agua puede calcularse como sigue: Una columna de agua de 1 pié de largo, 1 pié de ancho y 1 pié de alto (Volumen $=1$ pie $^{3}$; Figura 3) pesa $62.43 \mathrm{lb}$. Lo que significa que una columna de agua de 1 pie $^{3}$ dividida entre las 144 pulgadas $^{2}$ de la base tiene un peso de $0.43 \mathrm{lb}$ por cada pulgada ${ }^{2}$ (psi) (Tabla 2), una presión de $0.43 \mathrm{lb}$ por pulg².

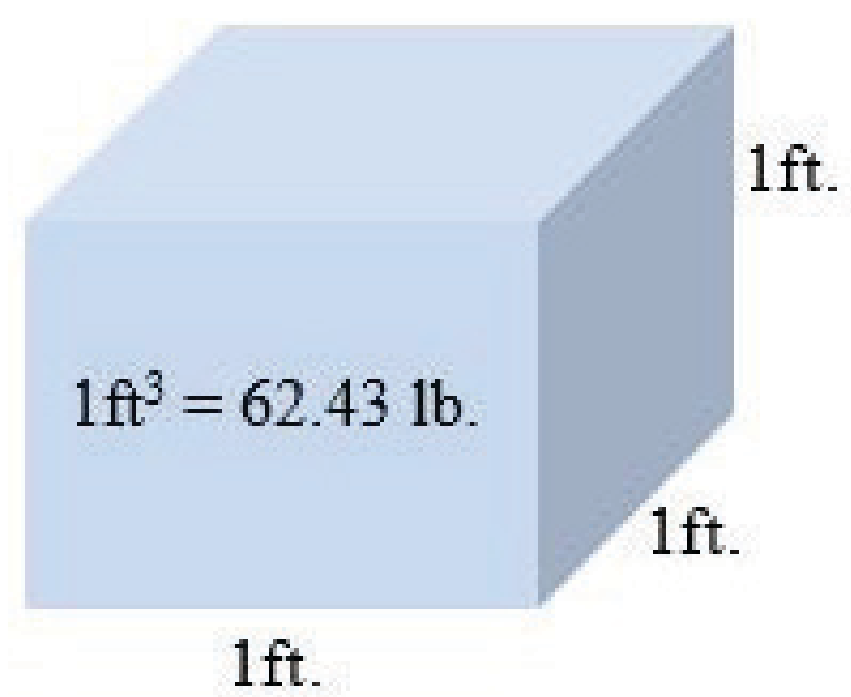

Figura 3. Ilustración de un volumen de 1 pié ${ }^{3}$ de agua. Créditos: Haimanote K. Bayabil, UF/IFAS

\section{Pérdidas por Fricción}

Al moverse el agua por el sistema de riego (p.e. tubería accesorios y válvulas, etc.) ésta pierde energía debido a la fricción del agua y del sistema. Si la presión aumenta la presión en el sistema baja, y parte del campo recibe menos agua de la asignada. Generalmente, tuberías de pequeño diámetro y más largas tienen más pérdidas por fricción comparado con tuberías de más diámetro y más cortas. Las pérdidas por fricción se tienen que considerar al diseñar los sistemas de riego para lograr una distribución uniforme y la aplicación deseada de agua. Factores como el diámetro de las tubería, tipo de material, y la forma, afectan las pérdidas por fricción. Las tablas de pérdidas por fricción se pueden usar para estimar pérdidas de presión en sistemas de riego. Por ejemplo, la Tabla 3 muestra las pérdidas por fricción para tuberías de goteo. Por ejemplo, en la Tabla 3 una tubería de goteo con un diámetro interno de 0.62 pulgadas y una descarga de 2 gpm tiene una pérdida por fricción de 1.76 psi por cada 100 pies de largo. La instalación de metros de presión puede ayudar a monitorear las pérdidas de presión de los sistemas de riego. Multiples metros de presión se pueden instalar en diferentes puntos del sistema para medir presiones diferentes.

\section{Caudal y Tamaño de Tubería}

El caudal en sistemas de riego depende del tamaño de la tubería y la presión. La cantidad de agua corriendo por la tubería está basada en qué cantidad de agua es descargada por los aspersores a lo largo de esa sección de tubería, capacidad de la bomba, y la presión. La velocidad es el caudal dividido por el área de la tuberia. Los tamaños de tuberia son mayores cerca del origen del agua y disminuyen hacia el final del regadío. El cálculo del tamaño correcto de la tubería asegurará la aplicación correcta del agua 
del riego. La Tabla 4 da el promedio de los caudales que distintos tamaños de tuberías pueden acomodar.

La Figura 4 enseña un sistema de aspersión con 5 aspersores; cada uno tiene un caudal de 4 gpm (equivalente da $20 \mathrm{gpm}$ para todo el sistema). Note la diferencia en los tamaños de las tuberías basadas en el total de agua corriendo en cada sección del sistema de riego (Tabla 4). Este ejemplo no considera pérdidas por ricción causadas por las características de la tubería (p.e. tipo, diámetro, longitud, accesorios, metro, etc.).
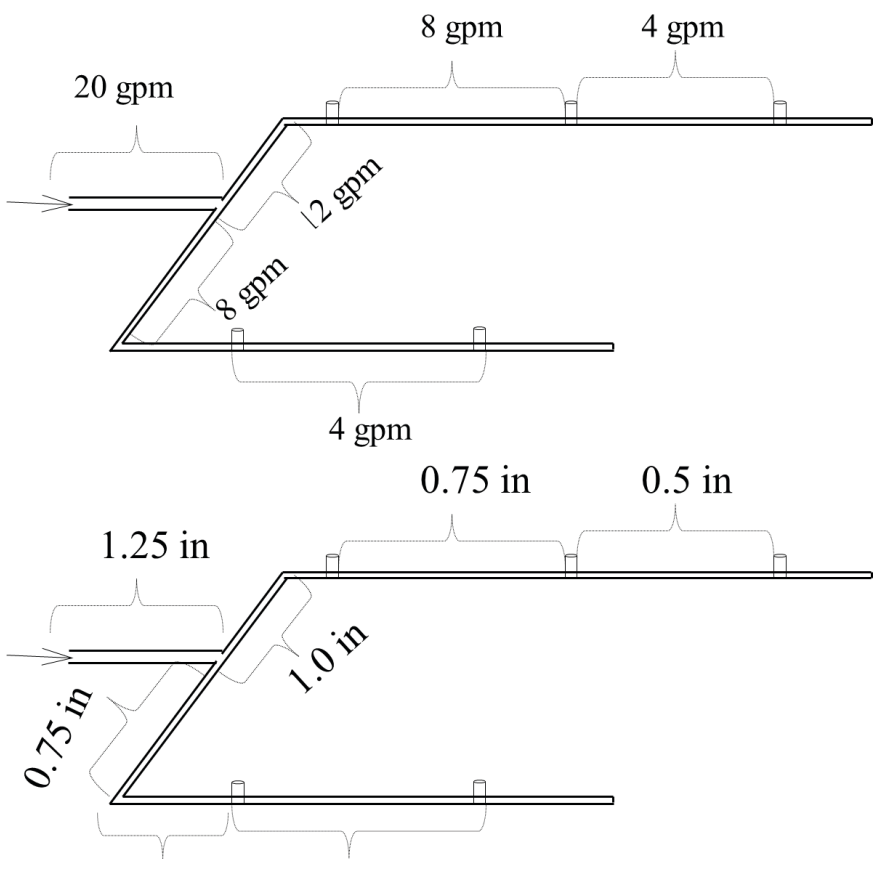
0.75 in
0.5 in

Figura 4. Esquema del diseño de riego por aspersión con caudales y tamaños de tuberías.

Créditos: Haimanote K. Bayabil, UF/IFAS

\section{Bombas de Regadío}

Las bombas se necesitan cuando el origen del agua está lejos del campo, y el riego es necesario de puntos de elevación de más bajos a más altos. La bomba se usa para crear flujo, elevar una presión que lleva a la adecuada corriente de agua en un regadío (Hanan 2017). La corriente requerida (gpm) y la presión del sistema de riego hay que considerarlas para seleccionar la bomba. Las bombas de riego pueden ser costosas. Considere el uso de su sistema de riego al seleccionar una bomba y, si es necesario, consulte a un profesional. Hanan (2017) y Hanan and Zazueta (2017) dan información adicional sobre tipos de bombas y medidas de eficiencia.

\section{Horario de Riego}

Las prácticas de riego principalmente suplementan los requerimientos de agua de las cosechas que no son satisfechas por la lluvia (Migliaccio and Li 2018). Los horarios de riego se refieren a cuando y cuanto riego es necesitado (Davis and Dukes 2010; Kiesekka et al. 2019). Los horarios de riego se pueden determinar usando medidas basadas en humedad del suelo, evapotranspiración y requerimiento de la cosecha. El diseño de sistemas de riego eficientes no garantiza el uso eficiente del agua a no ser que el apropiado horario de riego esté hecho. Información adicional sobre técnicas para horarios de riego se encuentra en los siguientes documentos en EDIS.

- Smart Irrigation Controllers: Operation of Evapotranspiration-Based Controllers: https://edis.ifas.ufl.edu/ae446

- Smart Irrigation Controllers: Programming Guidelines for Evapotranspiration-Based Irrigation Controllers: https:// edis.ifas.ufl.edu/ae445

- Evapotranspiration-Based Irrigation for Agriculture: Sources of Evapotranspiration Data for Irrigation Scheduling in Florida: https://edis.ifas.ufl.edu/ae455

- Evapotranspiration-Based Irrigation for Agriculture: Crop Coefficients of Some Commercial Crops in Florida: https:// edis.ifas.ufl.edu/ae456

- Evapotranspiration-Based Irrigation Scheduling for Agriculture: https://edis.ifas.ufl.edu/ae457

\section{Resumen}

Este documento provee información básica de los mayores factores que afectan el diseño de un sistema de riego. Varios factores incluyendo la selección de equipos apropiados y el diseño del sistema, influyen la efectividad de un sistema de riego. El diseño de eficientes sistemas de riego es crítico no sólo para conservar agua potable pero tambien para ahorrar dinero. Adicionalmente sistemas eficientes de regadio deben tener horarios de riego apropiados para obtener los resultados deseados.

\section{Referencias}

Cahn, M. D., and L. F. Johnson. 2017. "New Approaches to Irrigation Scheduling of Vegetables." Horticulturae 3:28.

Davis, S. L., and M. D. Dukes. 2010. "Irrigation Scheduling Performance by Evapotranspiration-Based Controllers." Agricultural Water Management 98:19-28. 
Dukes, M. D. 2012. "Water Conservation Potential of Landscape Irrigation Smart Controllers." Transactions of the ASABE 55:563-569.

Haman, D. Z. 2017. Pumps for Florida Irrigation and Drainage Systems. CIR832. Gainesville: University of Florida Institute of Food and Agricultural Sciences. https://edis.ifas. ufl.edu/wi001

Haman, D. Z., and F. S. Zazueta. 2017. Measuring Pump Capacity for Irrigation System Design. CIR1133. Gainesville: University of Florida Institute of Food and Agricultural Sciences. https://edis.ifas.ufl.edu/ae067

Irrigation Association. 2008. "Friction Loss Charts." http:// extension.missouri.edu/webster/documents/resources/ water/Friction_Loss_Charts-IrrigationAssociation.pdf

Kisekka, I., K. W. Migliaccio, M. D. Dukes, B. Schaffer, J. H. Crane, H. K. Bayabil, and S. M. Guzman. 2019. Evapotranspiration-Based Irrigation Scheduling for Agriculture. AE457. Gainesville: University of Florida Institute of Food and Agricultural Sciences. https://edis.ifas.ufl.edu/ae457

Marella, R. 2013. "Water Use in Florida, 2005 and Trends 1950-2005." U.S. Department of the Interior, U.S. Geological Survey. http://pubs.usgs.gov/fs/2008/3080/index.html

Marella, R. L. 2015. "Water Withdrawals in Florida, 2012: Open-File Report 2015-1156.” http://dx.doi.org/10.3133/ ofr20151156

Melby, P. 1988. Simplified Irrigation Design. PDA Publishers.

Migliaccio, K. W., and Y. C. Li. 2018. Irrigation Scheduling for Tropical Fruit Groves in South Florida. TR001. Gainesville: University of Florida Institute of Food and Agricultural Sciences. https://edis.ifas.ufl.edu/tr001

Rayer, S., and Y. Wang. 2017. "Projections of Florida Population by County, 2020-2045, with Estimates for 2016." Bureau of Economic and Business Research 50.

Rogers, J., T. Borisova, J. Ullman, K. T. Morgan, L. Zotarelli, and K. Gorgan. 2018. Factors Affecting the Choice of Irrigation Systems for Florida Tomato Production. FE960. Gainesville: University of Florida Institute of Food and Agricultural Sciences. https://edis.ifas.ufl.edu/fe960

Vico, G., and N. A. Brunsell. 2018. “Tradeoffs between Water Requirements and Yield Stability in Annual vs. Perennial Crops." Advances in Water Resources 112:189-202.
Zotarelli, L., L. Rens, C. Barrett, D. J. Cantliffe, M. D. Dukes, M. Clark, and S. Lands. 2019. Subsurface Drip Irrigation (SDI) for Enhanced Water Distribution: SDI-Seepage Hybrid System. HS1217. Gainesville: University of Florida Institute of Food and Agricultural Sciences. https://edis.ifas. ufl.edu/hs1217 
Tabla 1. Capacidad de retención de agua (pulgadas de agua por pie de profundidad de suelo) para varios tipos de suelos conunes en el sur de Florida (Migliaccio y Li 2018).

\begin{tabular}{|l|c|c|}
\hline \multirow{2}{*}{} & Soil & \multicolumn{2}{c}{ Capacidad Retención de Agua (CRA) } \\
\cline { 2 - 3 } & Rango (pulg/ft) & Promedio (pulg/ft) \\
\hline Pedregoso-limoso & $1.0-1.4$ & 1.2 \\
\hline Organo arcilloso & $1.2-2.4$ & 1.8 \\
\hline Turba & $2.0-3.0$ & 2.5 \\
\hline Arena y arena fina & $0.4-1.0$ & 0.75 \\
\hline
\end{tabular}

Tabla 2. Conversión de unidades de presión.

\begin{tabular}{|c|c|c|c|c|c|}
\hline & atm & in & bar & kPa & 101.32 \\
\hline atm & 1.0 & 406.79 & 1.013 & 0.249 \\
\hline in & 0.002456 & 1.0 & 0.00249 & 100 \\
\hline bar & 0.9872 & 401.47 & 1.0 & 1.03613 \\
\hline $\mathbf{k P a}$ & 0.00987 & 4.018 & 0.01 & 0.145 \\
\hline $\mathbf{p s i}\left(\mathbf{l b} / \mathbf{i n}^{2}\right)$ & 0.068 & 27.71 & 0.0689 & 0.00689 \\
\hline
\end{tabular}




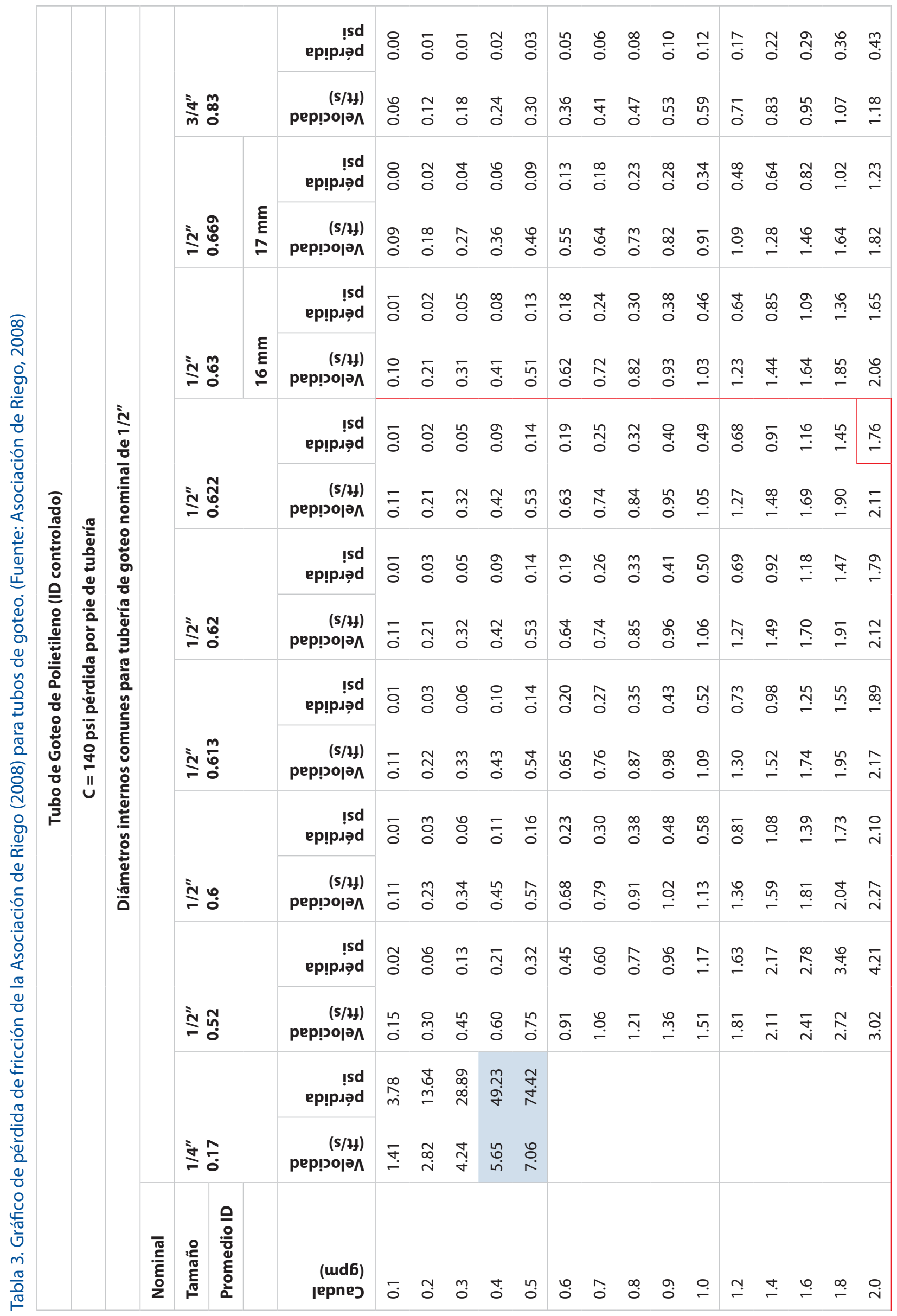




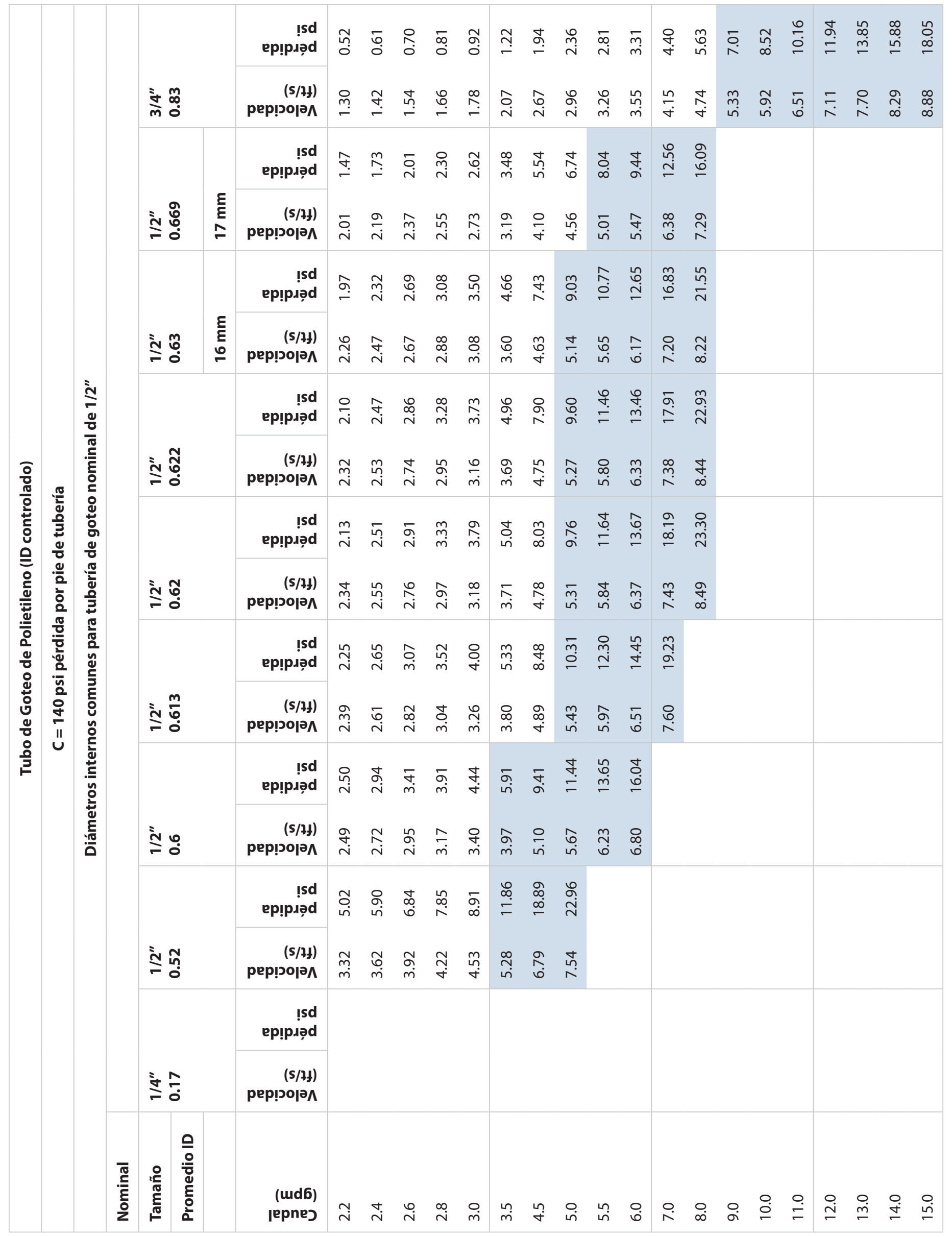


Tabla 4. Caudales ideales para tuberías de distintos tamaños (Origen: Melby, 1988).

\begin{tabular}{|c|c|}
\hline Tamaño de tubería (in) & Caudal (gpm) \\
\hline 0.5 & $1-6$ \\
\hline 0.75 & $7-10$ \\
\hline 1.0 & $11-16$ \\
\hline 1.25 & $17-26$ \\
\hline 1.5 & $27-35$ \\
\hline 2.0 & $36-55$ \\
\hline
\end{tabular}

